Supporting information for

\title{
HgTe Nanocrystals for SWIR Detection and their Integration up to Focal Plane Array
}

Audrey Chu ${ }^{1,2}$, Bertille Martinez ${ }^{1,3}$, Simon Ferre ${ }^{4}$, Vincent Noguier ${ }^{4}$, Charlie Gréboval ${ }^{1}$, Clément Livache ${ }^{1,3}$, Junling $\mathrm{Qu}^{1}$, Yoann Prado ${ }^{1}$, Nicolas Casaretto ${ }^{1}$, Nicolas Goubet ${ }^{1,5}$, Hervé Crugue $^{1}$, Lenart Dudy ${ }^{6}$, Mathieu G. Silly ${ }^{6}$, Grégory Vincent ${ }^{2}$, Emmanuel Lhuillier ${ }^{1 *}$

${ }^{1}$ Sorbonne Université, CNRS, Institut des NanoSciences de Paris, INSP, F-75005 Paris, France

2 ONERA-The French Aerospace Lab, Chemin de la Hunière, BP 80100, F-91123 Palaiseau, France

${ }^{3}$ Laboratoire de Physique et d'Étude des Matériaux, ESPCI Paris PSL Research University, Sorbonne Université Univ Paris 06, CNRS, 10 rue Vauquelin 75005 Paris, France.

${ }^{4} \mathrm{New}$ Imaging Technologies SA, 1 impasse de la Noisette 91370 Verrières le Buisson, France

${ }^{5}$ Sorbonne Université, CNRS, De la Molécule aux Nano-objets: Réactivité, Interactions et Spectroscopies, MONARIS, F-75005 Paris, France

${ }^{6}$ Synchrotron-SOLEIL, Saint-Aubin, BP48, F91192 Gif sur Yvette Cedex, France

*To whom correspondence should be sent: el@insp.upmc.fr 


\section{Table of content}

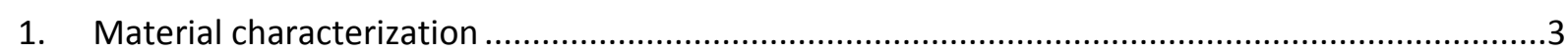

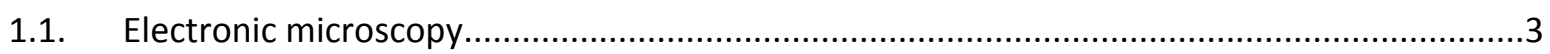

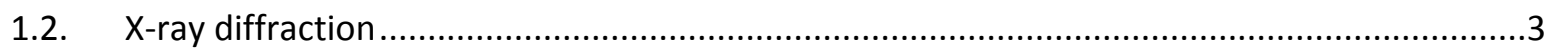

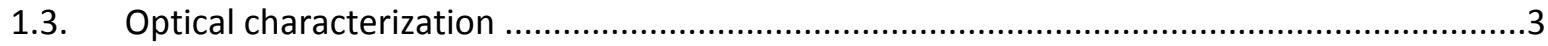

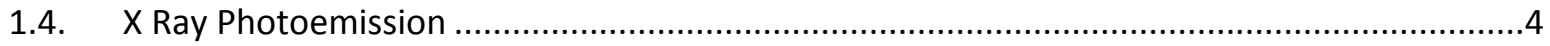

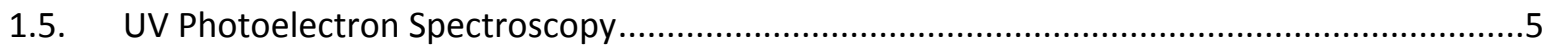

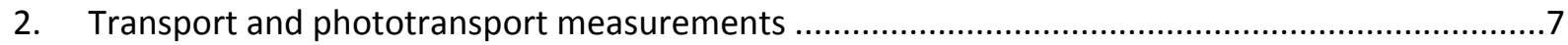

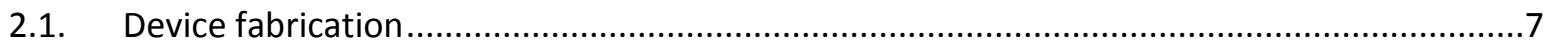

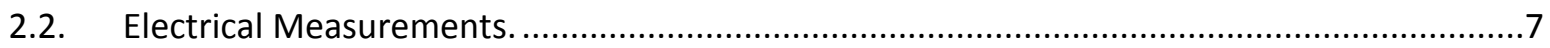

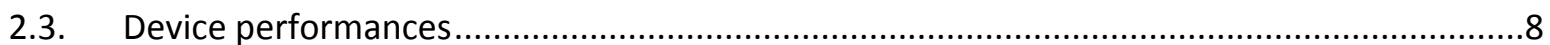

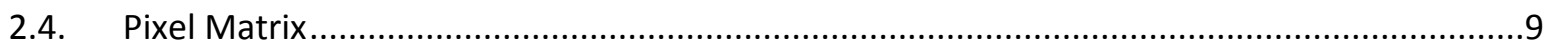

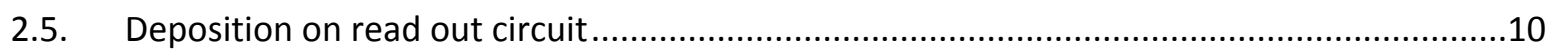

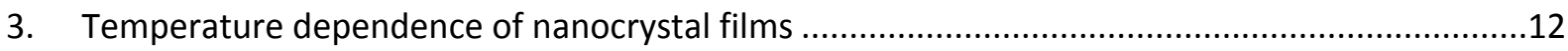

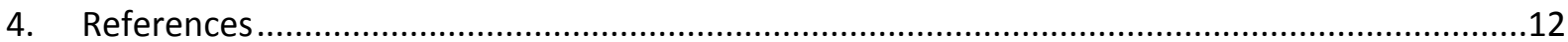




\section{Material characterization}

\subsection{Electronic microscopy}

For Transmission electronic microscopy (TEM) pictures, a drop of CQD solution is drop-cast on a copper grid covered with an amorphous carbon film. JEOL $2010 \mathrm{~F}$ is used at $200 \mathrm{kV}$ for acquisition of picture. For device characterization, a FEI Magellan scanning electron microscope is used.

For scanning electronic microscopy (SEM) pictures, FEI Magellan scanning electron microscope is used. The current of the beam is adjusted to $6 \mathrm{pA}$ and the operating bias is set to $3 \mathrm{kV}$.

\subsection{X-ray diffraction}

$\mathrm{X}$ ray diffraction is conducted by dropcasting a solution of CQDs on a Si wafer. The diffractometer is a Rigaku Smartlab based on the emission of the $\mathrm{Cu} \mathrm{K}_{\alpha}$ line. X-ray diffraction of the two materials reveals a zinc blende phase for $\mathrm{HgTe}^{1,2}$ and a rock slat phase for $\mathrm{PbS}^{3}$, see Figure $\mathrm{S} 1$
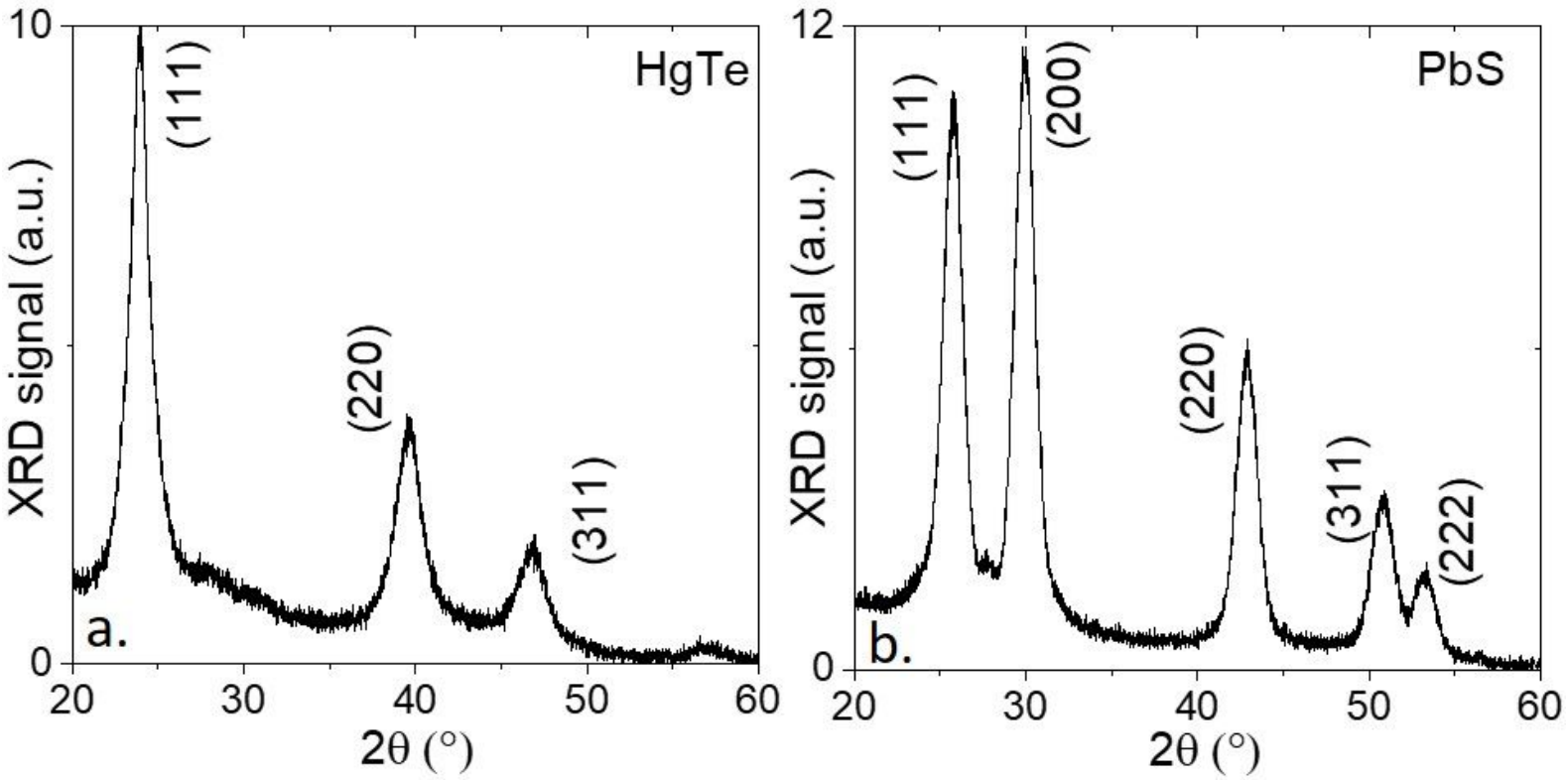

Figure $S 1$-ray diffractogram from HgTe (a.) and PbS (b.) CQD with a band edge at $6000 \mathrm{~cm}^{-1}$.

\subsection{Optical characterization}

Sample preparation for infrared spectroscopy. A glass slide is cut in $15 \times 15 \mathrm{~mm}^{2}$ pieces. Those substrates are rinsed with acetone and sonicated in acetone for 5 minutes. They are then rinsed with acetone and isopropanol and dried with a $\mathrm{N}_{2}$ gun. A solution of CQDs at $30 \mathrm{mg} \cdot \mathrm{mL}^{-1}$ in toluene is spincoated on those substrates ( $2000 \mathrm{rpm}, 60 \mathrm{~s}$ ). Afterwards, the ligand exchange procedure is carried out by dipping the film in the solution containing the new ligands for $60 \mathrm{~s}$ and rinsed in EtOH for $30 \mathrm{~s}$. All ligand exchange solutions are prepared at $1 \mathrm{w} \%$ in ethanol except $\mathrm{NH}_{4} \mathrm{Cl}$, for which the solvent is $\mathrm{MeOH}$. This process is repeated at least 2 to 3 times to build thick films.

Infrared spectroscopy is conducted using a Fisher IS50 Fourier transform Infrared spectrometer. The visible light source is used and shone on $\mathrm{CaF}_{2}$ beam splitter. To measure film absorption, an extended 
InGaAs detector is used. Each spectrum is averaged 32 times and the resolution is set to $4 \mathrm{~cm}^{-1}$. Photocurrent spectrum are acquired as the sample is biased using a Femto DLPCA current amplifier which role is also to magnify the current. The signal is then fed into the FTIR acquisition board.

Analysis of surface chemistry (Figure 1b): We observe the interband edge transition around $6000 \mathrm{~cm}^{-1}$. In addition, we observe a clear strong contribution from the $\mathrm{C}-\mathrm{H}$ bond at $2900 \mathrm{~cm}^{-1}$ relative to the ligand. There is a small peak from $\mathrm{CO}_{2}$ due to in air measurement. For PbS, we also observe a clear strong contribution from $\mathrm{C}=0$ bond due to the presence of oleate.

\subsection{Ray Photoemission}

Sample for XPS or UPS. Silicon wafer were rinsed with acetone, sonicated in acetone for 5 minutes. They were rinsed again with acetone and isopropanol and dried with $\mathrm{N}_{2}$ gun. A $5 \mathrm{~nm}$ layer of $\mathrm{Cr}$ and an $80 \mathrm{~nm}$ layer of Au were deposited using thermal evaporation. A thin layer ( $\approx 50 \mathrm{~nm}$ ) of QDs was deposited on those substrates using the same procedure described for photoconductive device preparation. Ligand exchange is conducted according to the same procedure. All ligand exchange solutions are prepared at $1 \mathrm{wt} \%$ in ethanol except $\mathrm{NH}_{4} \mathrm{Cl}$, for which the solvent is $\mathrm{MeOH}$.

The film is then introduced in the preparation chamber, degassed for at least an hour and transferred to the analysis chamber. The measurements were conducted at $600 \mathrm{eV}$. This photon energy is precisely measured using the first and second order of $4 \mathrm{f}$ core level of Au using the formula: $h v_{\text {exp }}=K E_{2^{n d}}-$ $K E_{1^{s t}}$.

The work function of the analyzer $\left(\mathrm{WF}_{\mathrm{A}}\right)$ is determined by measuring the kinetic energy of electrons at the Fermi level for the gold (set to $0 \mathrm{eV}$ ). $W F_{A}=h v_{\text {exp }}-K E_{F e r m i}$

For the measurement of the secondary electron cut-off, the sample is biased with an $18 \mathrm{~V}$ battery. The exact bias is determined by measuring the shift of the $4 \mathrm{f}$ core level of the gold when the bias is applied.

XPS overview. The overview is acquired with a $50 \mathrm{eV}$ pass energy and a $0.5 \mathrm{eV}$ resolution. The obtained spectra for $\mathrm{PbS}$ and $\mathrm{HgTe}$ are given in Figure $\mathrm{S} 2$.
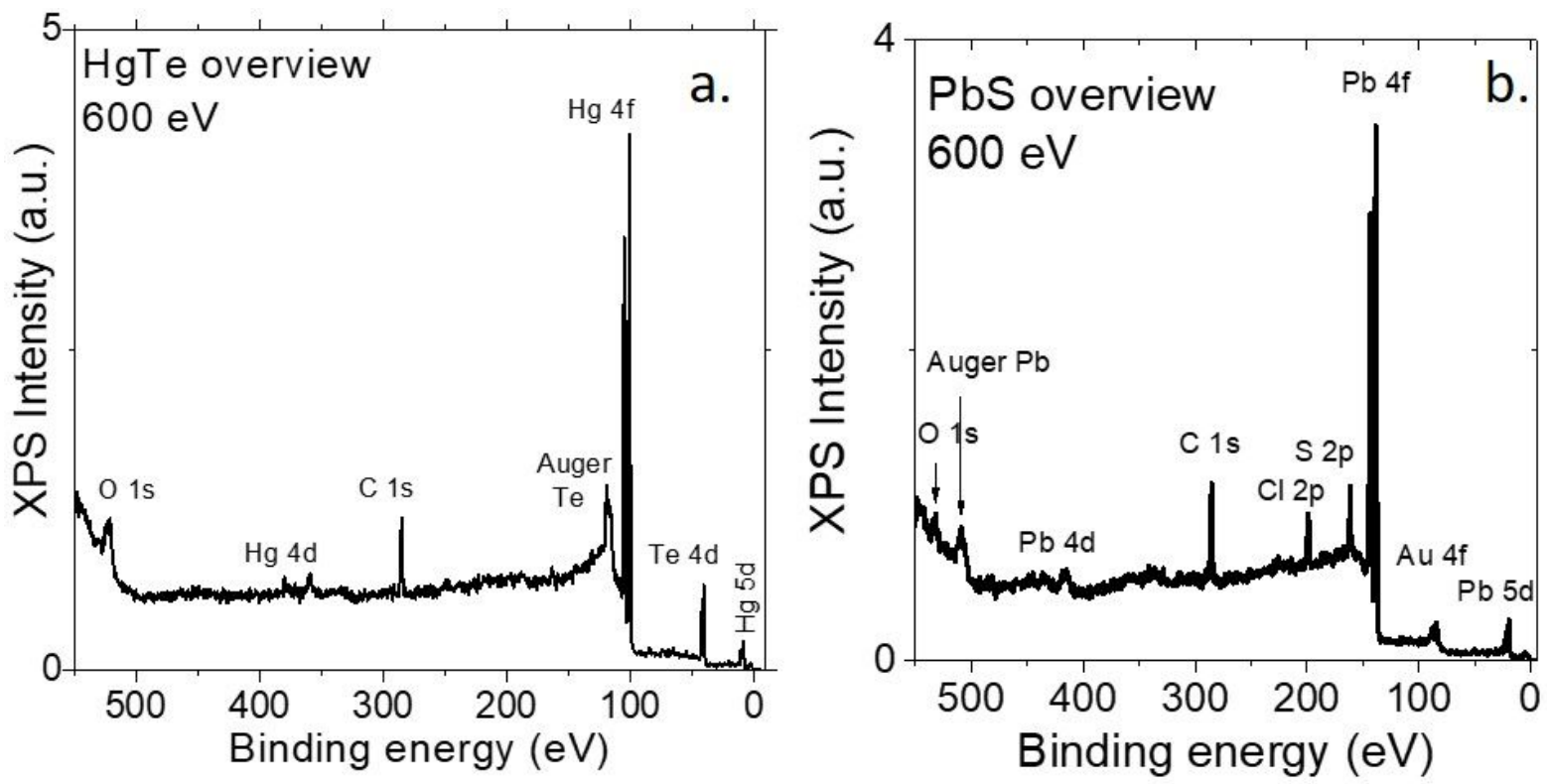

Figure S 2: XPS overview for a thin film of HgTe (a) or PbS (b) capped with EDT. 
In the case of HgTe we only observe contribution of $\mathrm{Hg}$, Te and from the ligands (C). A very limited $\mathrm{O}$ contribution is observed due to air preparation, see Figure $\mathrm{S} 2 \mathrm{a}$.

For $\mathrm{PbS}$, We notice contributions of $\mathrm{Pb}$ and $\mathrm{S}$, as well carbon and residual $\mathrm{Cl}$ from the $\mathrm{PbCl}_{2}$ precursor, see Figure $\mathrm{S} 2 \mathrm{~b}$.

Valence band measurement. We determine the value of $\mathrm{VB}-\mathrm{E}_{\mathrm{F}}$ looking at high KE electrons. We measure the highest kinetic energy available $\left(\mathrm{KE}_{\mathrm{VB}}\right)$ and extract $\mathrm{VB}-\mathrm{E}_{\mathrm{F}}$ with the formula: $V B-E_{F}=h$ $v_{\text {exp }}-K E_{V B}-W F_{A}$.

Work function measurement. In order to measure the work function, which is the difference in energy between vacuum level and Fermi level, we look for the cut off of secondary electrons ( $\left.\mathrm{KE}_{\text {cut off }}\right)$. We

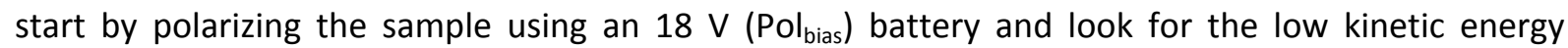
electrons. The work function is deduced with the formula: $W F_{\text {Sample }}=K E_{\text {Cut off }}-$ Pol $_{\text {Bias }}$.

\subsection{UV Photoelectron Spectroscopy}

The film is introduced in the preparation chamber and degassed for at least an hour and transferred to the analysis chamber. The measurements were conducted using a helium lamp. The photon energy is $21.21 \mathrm{eV}$.

The work function of the analyzer $\left(\mathrm{WF}_{\mathrm{A}}\right)$ is determined by measuring the kinetic energy of electrons at the fermi level for the gold (set to $0 \mathrm{eV}$ ). $W F_{A}=h v_{\text {exp }}-K E_{F e r m i}$

For the measurement of the secondary electron cut-off, the sample is biased with an $18 \mathrm{~V}$ battery. The exact bias is determined by measuring the shift of the $4 \mathrm{f}$ core level of the gold when the bias is applied.

Valence band measurement. We determine the value of $\mathrm{VB}-\mathrm{E}_{\mathrm{F}}$ looking at high $\mathrm{KE}$ electrons. We measure the highest kinetic energy available $\left(\mathrm{KE}_{\mathrm{VB}}\right)$ and extract $\mathrm{VB}-\mathrm{E}_{\mathrm{F}}$ with the formula: $V B-E_{F}=h$ $v_{\text {exp }}-K E_{V B}-W F_{A}$.

Work function measurement. In order to measure the work function, which is the difference in energy between vacuum level and Fermi level, we look for the cut off of secondary electrons. We start by polarizing the sample using an $18 \mathrm{~V}$ battery and look for the low kinetic energy electrons. The work function is deduced with the formula: $W F_{\text {Sample }}=K E_{\text {Cut off }}-$ Pol $_{\text {Bias }}$.

Figure S 3 shows the valence band and the secondary electron cut off for thin film of HgTe nanocrystals capped with various ligands. 

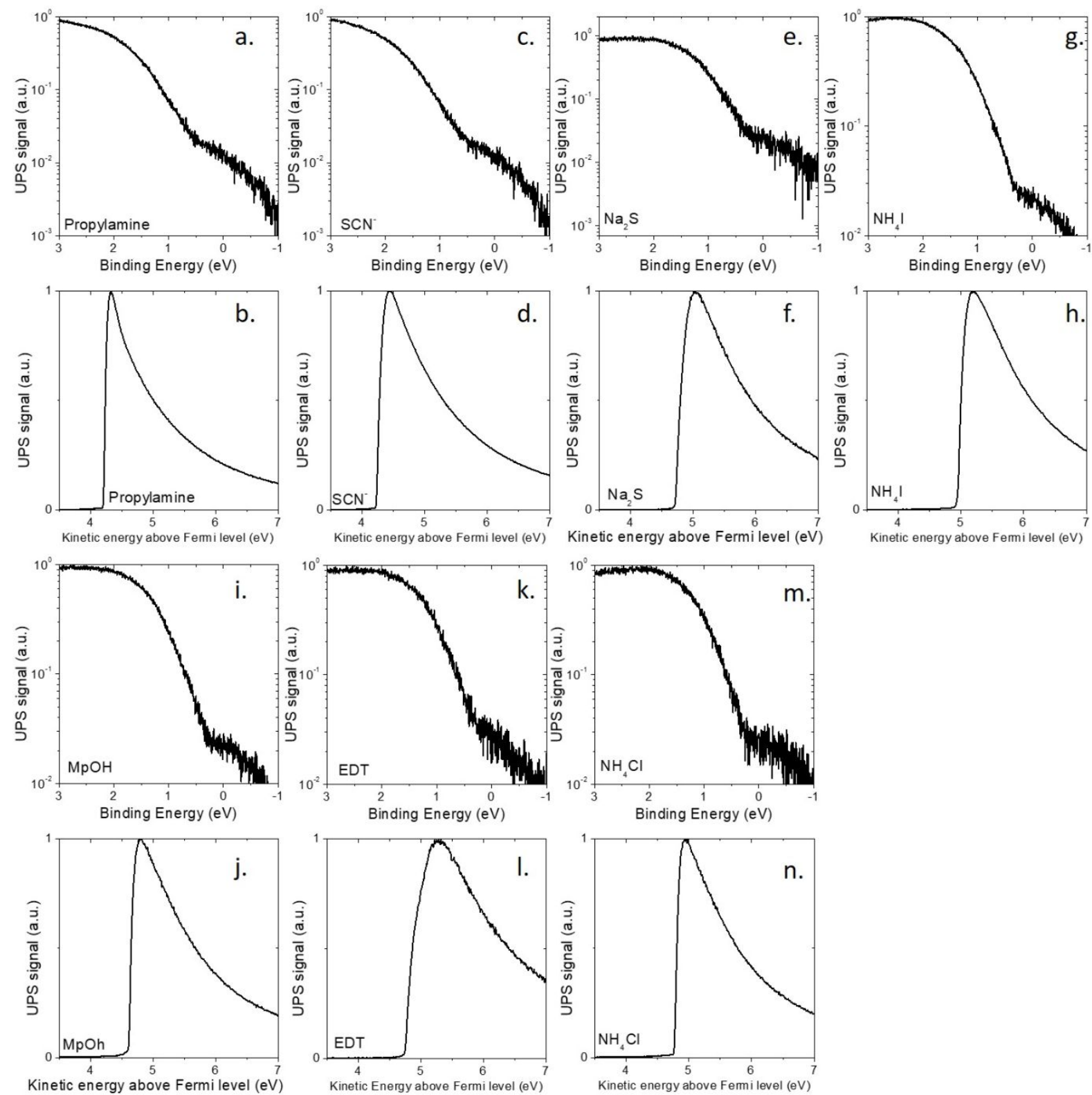

Figure S 3: Valence band signal as a function of the electron binding energy for a thin film of HgTe CQDs capped with propylamine (a), $\mathrm{SCN}^{-}(c), \mathrm{S}^{2-}(e), \mathrm{I}^{-}(\mathrm{g}), \mathrm{MpOH}(\mathrm{i}), \mathrm{EDT}(\mathrm{k})$ et $\mathrm{Cl}^{-}(\mathrm{m})$. Secondary electron cutoff as a function of the (re-calibrated) electron kinetic energy for a thin film of HgTe CQDs capped with propylamine (b), $\mathrm{SCN}^{-}(d), \mathrm{S}^{2-}(f), \mathrm{I}^{-}(\mathrm{h}), \mathrm{MpOH}(\mathrm{j}), \mathrm{EDT}(\mathrm{l})$ et $\mathrm{Cl}^{-}(\mathrm{n})$. The sample is polarized with an $18 \mathrm{~V}$ battery. 


\section{Transport and phototransport measurements}

\subsection{Device fabrication}

Electrolyte gating. In a $\mathrm{N}_{2}$ glovebox, $0.5 \mathrm{~g}$ of $\mathrm{LiClO}_{4}$ and $2.3 \mathrm{~g}$ of $\mathrm{PEG}$ are mixed in a vial. Thin vial is heated at $170{ }^{\circ} \mathrm{C}$ on a hot plate for $2 \mathrm{~h}$ until the solution becomes clear. To use the electrolyte, the solution is warmed up around $100{ }^{\circ} \mathrm{C}$ and brushed onto the CQD film.

$\mathrm{Si}_{\mathrm{SiO}} / \mathrm{Au}$ Electrodes. The surface of $\mathrm{Si} / \mathrm{SiO}_{2}$ wafer (400 $\mathrm{nm}$ oxide layer) is cleaned by sonication in acetone. The wafer is rinsed with acetone, then isopropanol and dried with a $\mathrm{N}_{2}$ gun. A final cleaning is made using an $\mathrm{O}_{2}$ plasma. An adhesion primer (TI PRIME) is spin-coated onto the substrate and baked at $120{ }^{\circ} \mathrm{C}$ for $2 \mathrm{~min}$. AZ5214E is spin-coated and baked at $110{ }^{\circ} \mathrm{C}$ for $90 \mathrm{~s}$. The substrate is exposed under UV through a pattern mask for $1.5 \mathrm{~s}$. The film is then baked at $125^{\circ} \mathrm{C}$ for 2 min in order to invert the resist. Then a $40 \mathrm{~s}$ flood exposure is performed. The resist is develop using a bath of AZ726 for 32 $\mathrm{s}$ and rinse in pure water and dried with $\mathrm{N}_{2}$. We then deposit $5 \mathrm{~nm}$ of chromium layer and $80 \mathrm{~nm}$ of gold layer using thermal evaporation. The lift-off is performed by dipping the film in acetone for 1 hour. The electrodes are rinsed using isopropanol and dried using a $\mathrm{N}_{2}$ gun. The electrodes are $2.5 \mathrm{~mm}$ long spaced by $20 \mu \mathrm{m}$. These electrodes are using for photoconductive devices and electrolyte-gated transistor measurements. A scheme of the transistor is given in Figure $\mathrm{S} 4$.

Photoconductive Device preparation. The solvent of CQD solution is changed for hexane-octane (9:1). Inside a $\mathrm{N}_{2}$ filled glovebox, $\mathrm{CQD}$ in hexane-octane are drop-casted on prepatterned interdigitated gold electrodes. After a complete drying, EDT ligand exchange is performed by dipping the film in an EDT solution in ethanol ( $1 \mathrm{wt} \%)$ for $90 \mathrm{~s}$ and rinsing it in pure ethanol for $30 \mathrm{~s}$. This process is repeated for 5-7 times to get homogeneous and crack free film with a device resistance of 1-2 $\mathrm{M} \Omega$.

\subsection{Electrical Measurements.}

DC Transport. The sample is connected to a Keithley 2634b, which control the drain bias ( $\left.\mathrm{V}_{\mathrm{DS}}\right)$ and measure the associated current $\left(\mathrm{I}_{\mathrm{DS}}\right)$. This measure is carried out in the dark or under illumination using $1.55 \mu \mathrm{m}$ diode.

Transistor Measurement. The sample is connected to a Keithley 2634b, which sets the drain source bias $\left(V_{D S}\right)$, control the gate bias $\left(V_{G S}\right)$ with a step of $1 \mathrm{mV}$ and measure the associated currents $\left(I_{D S}\right.$ and $\left.\mathrm{I}_{\mathrm{GS}}\right)$.

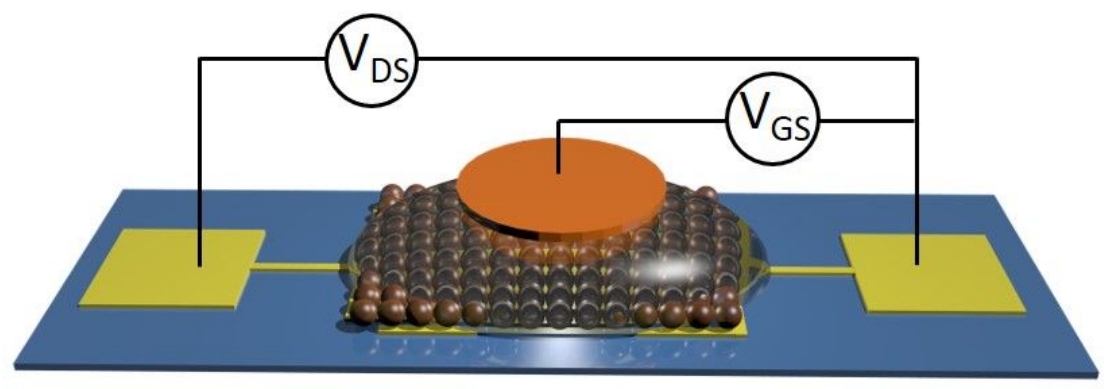

Figure $S 4$ : Scheme of an electrolyte transistor. 


\subsection{Device performances}

We have characterized the devices performances for both PbS and $\mathrm{HgTe}$, see Figure $\mathrm{S} 5$ and Figure $\mathrm{S} 6$ respectively. The detectivity is low for both materials: $2.6 \times 10^{7}$ Jones for PbS and $1.2 \times 10^{7}$ for $\mathrm{HgTe}$.
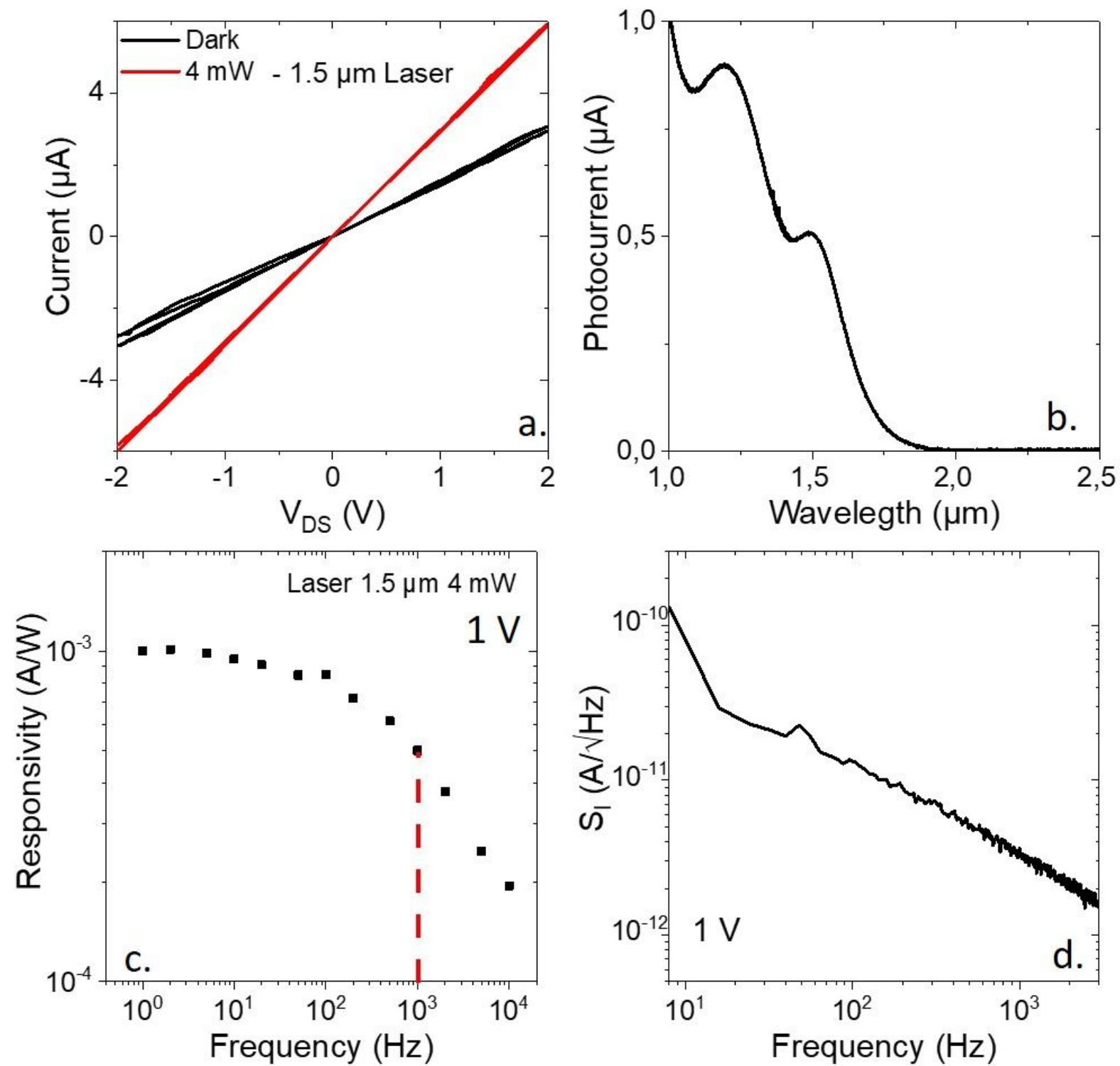

Figure S 5 PbS nanocrystals on interdigitated electrode : a. I-V curves under dark condition and under illumination $(\lambda=1.55 \mu \mathrm{m})$ measured at room temperature. b. Photocurrent spectra of the device. c. Responsivity at $1 \mathrm{~V}$ as a function of the signal frequency. $d$. Noise current spectral density as a function of the signal frequency. 

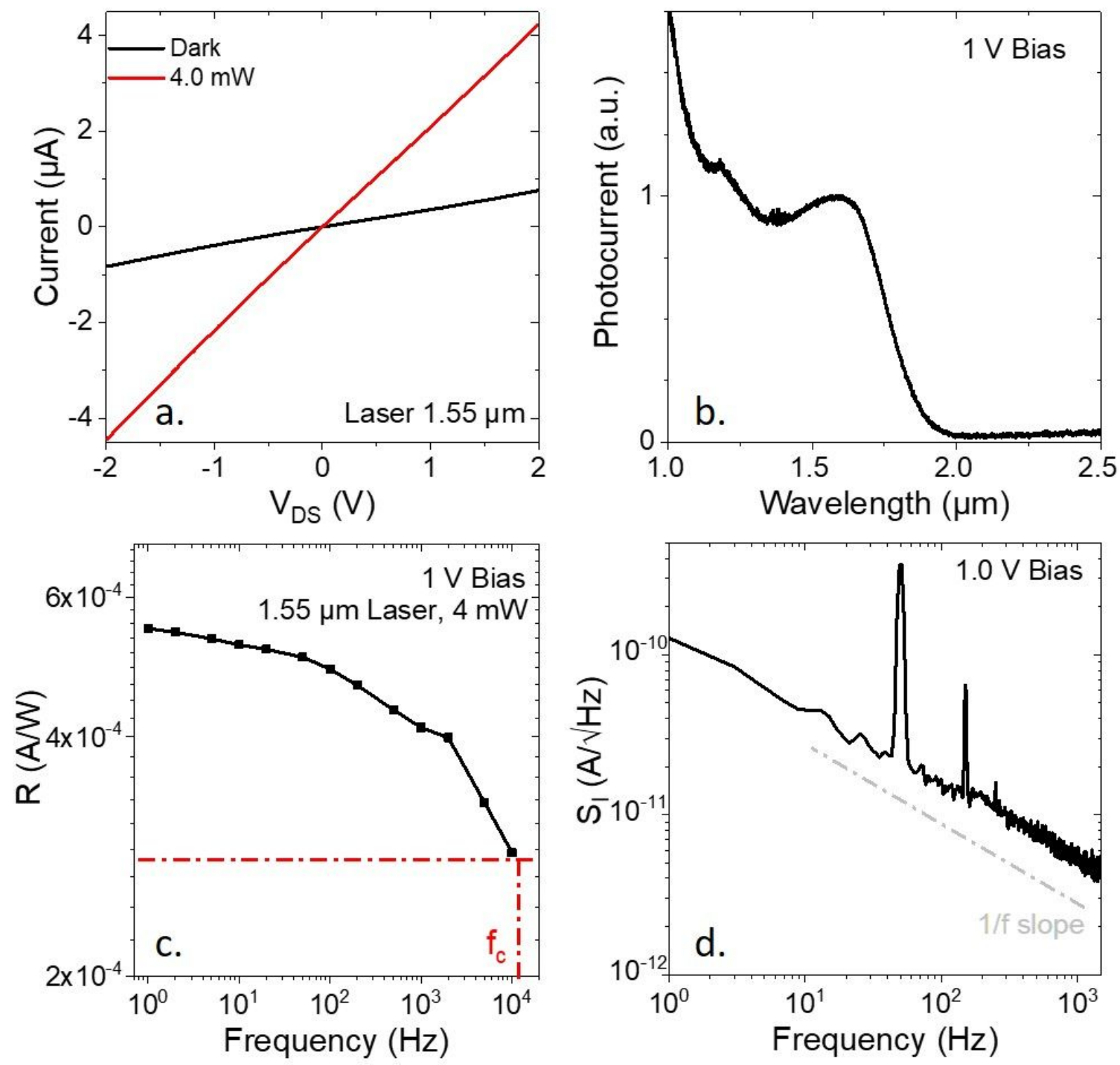

Figure S 6 HgTe nanocrystals on interdigitated electrode : a. I-V curves under dark condition and under illumination $(\lambda=1.55 \mu \mathrm{m})$ measured at room. $b$. Photocurrent spectra of the device. $c$. Responsivity at 1 $V$ as a function of the signal frequency. $d$. Noise current spectral density as a function of the signal frequency.

\subsection{Pixel Matrix}

The fabrication of the array of pixel is taken from Martinez et al. ${ }^{4}$ The matrix of pixel is made of $10 \times 10$ pixels with a $30 \mu \mathrm{m}$ size.

Electrode preparation. Step 1: A half microscope slide is washed with acetone, sonicated in an acetone bath for $4 \mathrm{~min}$ and then rinsed using acetone and isopropanol. It is then dried with $\mathrm{N}_{2}$ and plasmacleaned for $5 \mathrm{~min}$. Adhesion promoter (TI-PRIME) is spin coated on the substrate (4000 rpm, 1000 
$\mathrm{rpm} / \mathrm{s}, 30 \mathrm{~s}$ ) and then annealed at $120^{\circ} \mathrm{C}$ for $2 \mathrm{~min}$. The photosensitive resist (AZ 5214E) is then spin coated on the substrate $(4000 \mathrm{rpm}, 1000 \mathrm{rpm} / \mathrm{s}, 30 \mathrm{~s})$ and then annealed at $110^{\circ} \mathrm{C}$ for $90 \mathrm{~s}$. The sample is then exposed to UV through a first pattern chromium mask for $1.5 \mathrm{~s}$. The resist is inverted by an annealing step $\left(125^{\circ} \mathrm{C}, 2 \mathrm{~min}\right)$ and then a $40 \mathrm{~s}$ flood exposure is performed. The sample is developed in AZ 726 between 16 and $25 \mathrm{~s}$ and then rinsed in pure water. The patterned sample can then be plasma-cleaned again for 5 min before being put in a VINCl evaporator for a $5 \mathrm{~nm}$ of $\mathrm{Cr}$ and a $40 \mathrm{~nm}$ of gold depositions. At the end of evaporation, the sample is dipped in acetone overnight to remove the remaining resist. Step 2: The sample is washed with acetone and isopropanol before being dried with $\mathrm{N}_{2}$. The depositions of TI-PRIME and AZ 5214E resist follow the same procedure as described in step 1. The sample is then insolated through a second pattern chromium mask for $8 \mathrm{~s}$. This time, the lithography is positive so the resist is neither inverted with an annealing step neither re-exposed to UV for flood exposure. The sample is directly developed using AZ 726 developer for $45 \mathrm{~s}$ before being rinsed in pure water. The patterned sample is then put in an Alcatel sputtering chamber for a $27 \mathrm{~min}$ deposition of SiOx, at $120 \mathrm{~W}$. At the end of sputtering, the sample is dipped in acetone overnight to remove remaining resist, and finally sonicated at low power to gently remove the resist which is sticking on the substrate. The obtained SiOx layer is $75 \mathrm{~nm}$ thick. Step 3: After having rinsed the sample with acetone and isopropanol, the adhesion promoter and the resist are deposited as described in steps 2 and 3. The sample is then exposed through a third chromium mask for $1.5 \mathrm{~s}$. The same resist inversion process as described in step 1 (annealing + flood exposure) is performed before developing the sample in AZ 726 developer for $16 \mathrm{~s}$ and rinsing it in pure water. The patterned sample is then put in a $\mathrm{VINCl}$ evaporator for a $5 \mathrm{~nm} \mathrm{Cr}$ and a $150 \mathrm{~nm}$ Au depositions. At the end, the sample is dipped in acetone overnight to remove remaining resist.

Ink preparation. $30 \mathrm{mg}$ of $\mathrm{Na}_{2} \mathrm{~S}$ are dissolved in $10 \mathrm{~mL}$ of DMF. $0.5 \mathrm{~mL}$ of this short-ligand solution is added to $0.5 \mathrm{~mL}$ of a HgTe solution at $50 \mathrm{mg}^{\mathrm{mL}} \mathrm{m}^{-1}$ in toluene. A few $\mathrm{mL}$ of hexane can be added to help phase dissociation: the QDs migrate to the bottom phase (DMF), showing efficient ligand exchange. After 3 washing steps with hexane, the QDs are centrifuged at $6000 \mathrm{rpm}$ for $5 \mathrm{~min}$. The clear supernatant is discarded and the QDs are redispersed in $250 \mu \mathrm{L}$ of fresh DMF to reach a concentration of $100 \mathrm{mg} \cdot \mathrm{mL}^{-1}$. To remove all instable phase in the solution, it is centrifugated at $3000 \mathrm{rpm}$ for 3 minutes. The supernatant is the ink that we use. The ink is used immediately after preparation.

Ink deposition. The ink is deposited onto of the pixel matrix using spin-coating at $2000 \mathrm{rpm}$ for 1 minute. Seven layers are deposed to make the film.

\subsection{Deposition on read out circuit}

Ink preparation. $5 \mathrm{mg}$ of $\mathrm{HgCl}_{2}, 100 \mu \mathrm{L}$ of $\mathrm{MPOH}$ and $900 \mu \mathrm{L}$ of DMF are mixed. $0.5 \mathrm{~mL}$ of this solution is added to $0.5 \mathrm{~mL}$ of a HgTe solution at $50 \mathrm{mg}^{\mathrm{mL}}-1$ in toluene. A few mL of hexane can be added to help phase dissociation: the QDs migrate to the bottom phase (DMF), showing efficient ligand exchange. QDs are centrifuged at $6000 \mathrm{rpm}$ for $10 \mathrm{~min}$. The clear supernatant is discarded and the QDs are redispersed in DMF to reach a concentration of $300 \mathrm{mg}^{\mathrm{mL}}{ }^{-1}$. To ensure that the colloidal stability 
is good, the sample is then re-centrifuged at $3000 \mathrm{rpm}$ for $4 \mathrm{~min}$. If the ligand exchange is successful, no QDs should have fallen at the bottom of the tube at the end of centrifugation.

Ink deposition. The ink is deposited onto the read-out circuit using a two-steps spin-coating process: 1) at $600 \mathrm{rpm}\left(400 \mathrm{rpm} . \mathrm{s}^{-1}\right)$ for $180 \mathrm{~s}$ and 2$)$ at $3000 \mathrm{rpm}\left(1000 \mathrm{rpm} . \mathrm{s}^{-1}\right)$ for $30 \mathrm{~s}$. The film is then dried for at least 2 hours under primary vacuum. 


\section{Temperature dependence of nanocrystal films}

Optical and electrical properties of an HgTe film annealed for 15 minutes at different temperature $\left(50^{\circ} \mathrm{C}, 100^{\circ} \mathrm{C}\right.$ and $\left.150^{\circ} \mathrm{C}\right)$ are very similar if the baking is made under ambient atmosphere (Air) or under inert conditions in a $\mathrm{N}_{2}$ glovebox, see Figure $\mathrm{S} 7$. However, for PbS CQD film, the annealing under inert conditions is quite different than the one under air. Frist no blue shift is observed at $50^{\circ} \mathrm{C}$. Secondly at $150^{\circ} \mathrm{C}$ the optical feature is completely lost to obtain a long tail of absorption. Finally, the dark current is dramatically increased with annealing step under inert condition while it was stable under ambient condition. All those observations are consistent with the formation of a shell of $\mathrm{PbO}$ with an annealing in air.
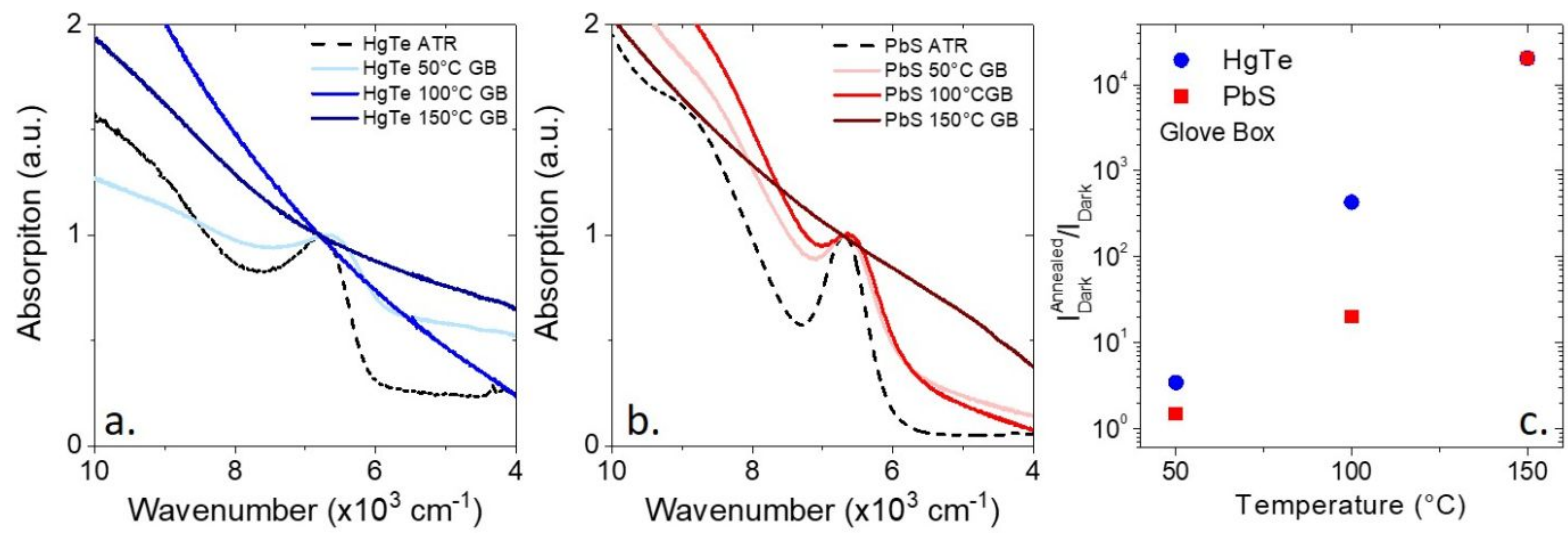

Figure S 7: a. Absorption of HgTe nanocrystals thin film in solution and under thin film form after annealing at various temperature in glove box. $b$. Absorption of PbS nanocrystals thin film in solution and under thin film form after annealing at various temperature in glove box. c. Ratio of the current after and before annealing for thin film of $\mathrm{HgTe}$ and PbS nanocrystal as a function of the annealing temperature. The annealing is made in a $\mathrm{N}_{2}$ filled glovebox.

\section{References}

(1) Keuleyan, S.; Lhuillier, E.; Guyot-Sionnest, P. Synthesis of Colloidal HgTe Quantum Dots for Narrow Mid-IR Emission and Detection. J. Am. Chem. Soc. 2011, 133, 16422-16424.

(2) Kovalenko, M. V.; Kaufmann, E.; Pachinger, D.; Roither, J.; Huber, M.; Stangl, J.; Hesser, G.; Schäffler, F.; Heiss, W. Colloidal HgTe Nanocrystals with Widely Tunable Narrow Band Gap Energies: From Telecommunications to Molecular Vibrations. J. Am. Chem. Soc. 2006, 128, 35163517.

(3) Moreels, I.; Lambert, K.; Smeets, D.; De Muynck, D.; Nollet, T.; Martins, J. C.; Vanhaecke, F.; Vantomme, A.; Delerue, C.; Allan, G.; et al. Size-Dependent Optical Properties of Colloidal PbS Quantum Dots. ACS Nano 2009, 3, 3023-3030.

(4) Martinez, B.; Ramade, J.; Livache, C.; Goubet, N.; Chu, A.; Gréboval, C.; Qu, J.; Watkins, W. L.; Becerra, L.; Dandeu, E.; et al. HgTe Nanocrystal Inks for Extended Short-Wave Infrared Detection. Advanced Optical Materials 2019, 1900348. 